\title{
Prevalência de disfunções sexuais no puerpério de parto cesáreo
}

\author{
Prevalence of sexual dysfunctions in the puerperium of cesarean delivery \\ Prevalencia de disfunciones sexuales en el puerperio del parto por cesárea
}

Thayna Maria Praia Baratella

ORCID: https://orcid.org/0000-0003-0890-7259 Universidade Católica de Pernambuco, Brasil

E-mail: thaynapraiafta@gmail.com

Ana Carolina da Silva Quiros

ORCID: https://orcid.org/0000-0001-6407-3282 Universidade Católica de Pernambuco, Brasil

E-mail: anacarolinaquiros@hotmail.com

Maria Eduarda Siqueira Veras

ORCID: https://orcid.org/0000-0001-9400-2987 Universidade Católica de Pernambuco, Brasil E-mail:dudasveras1@gmail.com

Jamile Maria de Souza Alves

ORCID: https://orcid.org/0000-0002-8132-0940 Universidade Católica de Pernambuco, Brasil E-mail: jamilesouzafisio@gmail.com

Thaynan Santos da Silva

ORCID: https://orcid.org/0000-0001-6879-2591 Universidade Católica de Pernambuco, Brasil

E-mail: thaynansantos10@hotmail.com

Marina de Lima Neves Barros

ORCID: https://orcid.org/0000-0002-3544-0538 Universidade Católica de Pernambuco, Brasil E-mail: marinalnbarros@gmail.com

Érica Patrícia Borba Lira Uchôa

ORCID: https://orcid.org/0000-0003-4099-1876

Universidade Católica de Pernambuco, Brasil E-mail: ericaluchoa@gmail.com

Valéria Conceição Passos de Carvalho

ORCID: https://orcid.org/0000-0001-8314-9000

Universidade Católica de Pernambuco, Brasil E-mail: valeriapassos@gmail.com

\begin{abstract}
Resumo
Objetivo: Descrever a prevalência de disfunções sexuais no puerpério de parto Cesário. Metodologia: Estudo do tipo observacional descritivo, o tamanho da amostra foi de 30 puérperas. Sendo desenvolvido de forma digital, através de um formulário criado no Google Forms de outubro de 2020 a abril de 2021. A primeira etapa contou com o preenchimento dos dados sociodemográficos no formulário e em seguida aplicação do Female Sexual Function Index (FSFI), um autorrelato com 19 itens que abrange seis domínios sexuais (desejo, excitação, lubrificação, orgasmo, satisfação e dor), nas últimas quatro semanas, a paciente seleciona uma das respostas que mais lhe representa, a alternativa 0 indica que não teve relação sexual e as outras variam de 1 a 5 . O somatório final reúne as respostas de todos os domínios. A análise estatística foi descritiva. Resultados: Dentre as voluntárias, $80 \%$ da população possui faixa etária entre 19-29 anos de idade e 43,3\% das mulheres são casadas, $53,3 \%$ das mulheres concluíram o ensino superior. $70 \%$ da amostra esteve grávida apenas 1 vez e 76,6\% realizaram apenas 1 cesárea até o momento da pesquisa. Em relação ao desejo, 36,7\% das mulheres relataram sentir desejo ou interesse sexual poucas vezes. Quando se questiona sobre a satisfação sexual, 53,3\% relataram estar muito satisfeitas com a intensidade de intimidade emocional com seu parceiro. Conclusão: O presente estudo evidenciou que as mulheres participantes da pesquisa que estavam no puerpério de parto Cesáreo não apresentaram uma DSF e sim uma alteração moderada na atividade sexual.
\end{abstract}

Palavras-chave: Período pós parto; Prevalência; Questionário; Sexualidade.

\begin{abstract}
Objective: To describe the prevalence of sexual dysfunctions in the postpartum period of Cesarean delivery. Methodology: Descriptive observational study, the sample size was 30 postpartum women. Being developed digitally, through a form created in Google Forms from October 2020 to April 2021. The first step included filling in the sociodemographic data on the form and then applying the Female Sexual Function Index (FSFI), a self-
\end{abstract}


report with 19 items covering six sexual domains (desire, arousal, lubrication, orgasm, satisfaction and pain), in the last four weeks, the patient selects one of the answers that best represents her, the alternative 0 indicates that she did not have sexual intercourse and the others range from 1 to 5 . The final sum gathers the responses from all domains. Statistical analysis was descriptive. Results: Among the volunteers, $80 \%$ of the population is aged between 19-29 years old and $43.3 \%$ of the women are married, 53.3\% of the women have completed higher education. $70 \%$ of the sample was pregnant only once and $76.6 \%$ had only 1 cesarean at the time of research. Regarding desire, $36.7 \%$ of women reported feeling sexual desire or interest a few times. When asked about sexual satisfaction, 53.3\% reported being very satisfied with the intensity of emotional intimacy with their partner. Conclusion: This study showed that women participating in the research who were in the postpartum period of Cesarean delivery did not present a DSF, but a moderate change in sexual activity.

Keywords: Postpartum period; Prevalence; Quis; Sexuality.

\section{Resumen}

Objetivo: Describir la prevalencia de disfunciones sexuales en el posparto del parto por cesárea. Metodología: Estudio observacional descriptivo, el tamaño de la muestra fue de 30 puérperas. Se está desarrollando digitalmente, a través de un formulario creado en Google Forms desde octubre de 2020 hasta abril de 2021. El primer paso consistió en completar los datos sociodemográficos en el formulario y luego aplicar el Índice de Función Sexual Femenina (FSFI), un autoinforme con 19 ítems que cubren seis dominios sexuales (deseo, excitación, lubricación, orgasmo, satisfacción y dolor), en las últimas cuatro semanas, la paciente selecciona una de las respuestas que mejor la representa, la alternativa 0 indica que no tuvo relaciones sexuales y las otras rango de 1 a 5. La suma final reúne las respuestas de todos los dominios. El análisis estadístico fue descriptivo. Resultados: Entre los voluntarios, el 80\% de la población tiene entre 19 y 29 años y el 43,3\% de las mujeres están casadas, el 53,3\% de las mujeres tiene estudios superiores completos. El 70\% de la muestra estaba embarazada solo una vez y el 76,6\% tenía solo 1 cesárea al momento de la investigación. En cuanto al deseo, el 36,7\% de las mujeres informó haber sentido deseo o interés sexual en algunas ocasiones. Cuando se les preguntó sobre la satisfacción sexual, el 53,3\% informó estar muy satisfecho con la intensidad de la intimidad emocional con su pareja. Conclusión: Este estudio mostró que las mujeres participantes en la investigación que se encontraban en el período posparto de parto por cesárea no presentaban FSD, sino un cambio moderado en la actividad sexual.

Palabras clave: Posparto; Predominio; Prueba; Sexualidad.

\section{Introdução}

Segundo a Organização Mundial de Saúde (OMS), a saúde sexual corresponde a um estado de saúde físico, emocional, mental e de bem-estar social em relação à sexualidade. A sexualidade, por sua vez, é um dos indicadores para qualidade de vida e recebe a influência de diversos fatores, tais como: Biológicos; Psicológicos; Socioeconômicos; Éticos e Espirituais, não se restringindo apenas à meta reprodutiva, mas também fortalece relações amorosas e afetivas entre as pessoas (Ministério da Saúde, 2013).

Ao tratarmos da sexualidade feminina pode se dizer que esta é organizada e coordenada em 4 fases: Desejo; Excitação; Orgasmo e Resolução. Quando alguns dos fatores relacionados a sexualidade são comprometidos, teremos um quadro de Disfunção Sexual Feminina (DSF), caracterizada por uma perturbação clinicamente significativa na capacidade de uma pessoa responder ou experimentar prazer sexual (Barreto et al., 2018). As DSF são definidas como comprometimento do desejo e da excitação sexual, do orgasmo e/ou dor sexual que provoquem desconforto pessoal significativo e que influenciam negativamente a qualidade de vida das mulheres acometidas, quanto a sua prevalência temos que aproximadamente 52\% a 45\% das mulheres relata algum tipo de insatisfação na sua vida sexual (Santos et al., 2019).

O puerpério corresponde à fase da vida da mulher em que todas as modificações fisiológicas e biomecânicas retornam ao estado pré-gravídico. Ele tem início com a saída da placenta e prolonga-se por 6 a 8 semanas, sendo dividido em três fases: Puerpério imediato ( $1^{\circ}$ ao $10^{\circ}$ dia); Puerpério tardio $\left(11^{\circ}\right.$ ao $45^{\circ}$ dia) e Puerpério remoto (a partir do $45^{\circ}$ dia). É importante ressaltar que é no puerpério imediato que ocorrem as mais importantes alterações fisiológicas, por exemplo, a involução uterina. As principais queixas relatadas pelas puérperas nessa fase são: dor relacionada às contrações uterinas, flatulências, desconfortos gastrointestinais, dores na cervical e lombar, queixas quanto à amamentação (dor, fissuras, mastites, 
ingurgitamento mamário), edemas, diástase do músculo reto abdominal, dor na incisão da Cesária, dor na episiotomia, incontinência urinária, depressão pós-parto e, além de tudo, a falta de orientações (Nunes et al., 2019).

A gravidez e puerpério são períodos da vida da mulher marcados por frequentes dificuldades sexuais. Inúmeros fatores interferem na função sexual nesse período, incluindo alterações hormonais, anatômicas, psicológicas e sociais (De Araújo et al., 2019). Na cesárea não há alteração no períneo para a saída do feto, mas todas as mudanças experimentadas durante a gestação podem ser somadas a outros fatores como desconforto na cicatriz, consequentemente, influenciando negativamente na função sexual. As taxas de Cesárea no Brasil estão aumentando, bem como, os seus efeitos (Pereira et al., 2018).

No puerpério, muitas mulheres continuam a relatar declínio no interesse ou desejo sexual, porém $80 \%$ dos casais restabelecem relações sexuais até a $12^{\mathrm{a}}$ semana após o parto. Aos seis meses pós-parto, a maioria das mulheres retornou à atividade sexual aos 12 meses, a maioria das mulheres considera a sua vida sexual semelhante ao período pré-gestacional (De Araújo et al., 2019). Sendo assim, o diagnóstico precoce das DSF ainda no puerpério, é importante para detectar conflitos emocionais, além de interferir diretamente na qualidade de vida e na saúde da mulher (Silva et al., 2017).

Diante do exposto, observa-se que as DSF no pós parto tornam-se ada vez maiores e menos tratadas durante este período. Sendo assim, surge a necessidade de melhorar o conhecimento dos profissionais que lidam com a saúde da mulher acerca das DSF presentes no período puerperal de uma Cesária, para que possam ser planejadas medidas de promoção a saúde e de prevenção destas disfunções e por conseguinte melhorar a qualidade de vida destas mulheres. Além de descrever a prevalência das disfunções sexuais no puerpério, o perfil sócio demográfico e reprodutivo dessas mulheres.

\section{Metodologia}

O estudo realizado está vinculado a Universidade Católica de Pernambuco, a Escola de Saúde e Ciências da Vida e ao curso de FISIOTERAPIA. Parte integrante do projeto de pesquisa intitulado "Perfil Epidemiológico, Avaliação, Tratamento E Prevenção Das Disfunções Do Assoalho Pélvico Em Adultos", cadastrado sob o CAAEE 02977018.4.0000.5206 encaminhado ao comitê de ética de pesquisa com seres humanos, obedecendo às orientações da Resolução 466/12 da Comissão Nacional de Ética em pesquisa, órgão do Ministério da Saúde e aprovado sob o parecer nº 3.049.835.

Estudo do tipo observacional descritivo, o tamanho da amostra por conveniência foi de 30 puérperas. Sendo desenvolvido de forma digital, através de um formulário criado no Google Forms e divulgado em redes de comunicação (instagram, facebook e whatsapp). Para compor a amostra as pacientes foram convidadas a participar deste trabalho voluntariamente e ao abrir o link da pesquisa já é possível ver uma página explicando todos os objetivos e benefícios do presente estudo, sendo solicitada a confirmação e o preenchimento do campo referente ao Termo de Consentimento Livre e Esclarecimento (TCLE).

Como Critérios de inclusão temos: puérperas de parto Cesário, na faixa etária entre 18-40 anos e com vida sexual ativa. Entre os critérios de exclusão temos: pacientes com deficiência cognitiva que impossibilite o entendimento entre pesquisada e pesquisador, puérperas que apresentem alguma patologia (vulvovaginites, infecção urinária) no assoalho pélvico e aquelas que não queiram participar da pesquisa.

$\mathrm{Na}$ fase inicial as puérperas foram abordadas no puepério, em ambiente virtual através de um post e um texto explicativo sobre a pesquisa e como se da o seu desenvolvimento. A segunda parte aconteceu quando a mulher aceitou participar da pesquisa e o link de preenchimento foi enviado para a mesma. A primeira seção do link da pesquisa trás uma explicação sobre os termos como o que é a sexualidade, quais as suas fases e sobre a DSF. Logo em seguida vem o TCLE com todas as normas bem explicadas para que a mulher se sinta assegurada das informações que vão ser prestadas. Na terceira seção do link está o questionário composto por perguntas estruturadas para obtenção do perfil sociodemográfico e reprodutivo 
das mulheres, ou seja, idade, raça, religião, escolaridade, profissão, renda mensal familiar, número de pessoas no domicílio, histórico reprodutivo, dentre outros. O questionário contém questões objetivas e foi elaborado pela pesquisadora para a obtenção dos objetivos estipulados.

$\mathrm{Na}$ última seção do link da pesquisa continha o questionário de avaliação da função sexual. Para este momento foi escolhido o Female Sexual Function Index (FSFI), um auto-relato com 19 itens que abrange seis domínios sexuais (desejo, excitação, lubrificação, orgasmo, satisfação e dor), nas últimas quatro semanas. A paciente seleciona em cada questão uma das seis alternativas possíveis que melhor descreve sua situação. A alternativa 0 indica que não teve relação sexual e as outras variam de 1 a 5 . A análise é realizada reunindo as respostas em seis domínios diferentes: desejo itens 1 e 2; excitação itens 3, 4, 5 e 6; lubrificação itens 7, 8, 9, e 10; orgasmo itens 11, 12 e 13; satisfação itens 14, 15 e 16; desconforto/dor itens 17, 18 e 19 (Barreto et al., 2018).

O questionário segue uma sequencia numérica e para obter o resultado final é necessário realizar a soma das pontuações dos itens individuais dessa dimensão e pela posterior multiplicação desse resultado por um valor específico de ponderação atribuído a cada dimensão que vai de 0,4 à 0,6 . O Score mínimo e máximo que se pode atingir por domínio varia de 0 a 6 e a pontuação total do FSFI varia entre 2 e 36, quanto maior a pontuação maior o nível de função sexual. O ponto de corte que demonstra uma boa função sexual é de >27,5; (DA Silva e Silas, 2019).

Tabela 1: Escore dos domínios do questionário FSFI.

\begin{tabular}{lccccc}
\hline \multicolumn{1}{c}{ Domínio } & Questão & Variação do escore & Fator & Escore mínimo & Escore máximo \\
\hline Desejo & 1,2 & $1-5$ & 0,6 & 1,2 & 6,0 \\
Excitação & $3,4,5,6$ & $0-5$ & 0,3 & 0 & 6,0 \\
Lubrificação & $7,8,9,10$ & $0-5$ & 0,3 & 0 & 6,0 \\
Orgasmo & $11,12,13$ & $1-5$ & 0,4 & 0 & 6,0 \\
Satisfação & $14,15,16$ & 0 (ou 1) -5 & 0,4 & 0,8 & 6,0 \\
Dor & $17,18,19$ & $0-5$ & 0,4 & 0 & 6,0 \\
Escore Total & & & & 2,00 & 36,0 \\
\hline
\end{tabular}

A tabela demonstra como calcular o resultado de cada domínio do questionário FSFI. Fonte: Autores (2021).

Nos resultados foi realizada uma análise descritiva, baseados na composição de tabelas de frequências ou gráficos.

\section{Resultados}

A amostra do estudo contou com 30 mulheres que participaram voluntariamente e preencheram de forma virtual o questionário criado sobre as questões sociodemográficas, perfil reprodutivo e o questionário Female Sexual Function Index (FSFI).

A Tabela 2 demonstra trás o perfil sociodemográfico das mulheres em puerpério imediato de Cesárea. Nesta, verificamos que 80\% da população possui faixa etária entre 19-29 anos de idade. Quanto a profissão 23,3\% são fisioterapeutas e 23,3\% enquadram-se em outras profissões como costureira, secretaria e outras. Sobre o estado civil 43,3\% das mulheres são casadas e 66,6\% moram na região metropolitana do Recife. Em relação a cor, 43,3\% relataram se considerar pardas e 50\% da população do estudo declarou ser católica. Quanto a escolaridade 53,3\% das mulheres concluíram o ensino superior. Sobre a prática de fumar $96,7 \%$ da amostra informou não fumar, $60 \%$ relataram não ingerir bebida alcoólica e 73,3\% não prática nenhuma atividade física. Todos os cálculos foram feitos com $n=30$. 
Research, Society and Development, v. 10, n. 9, e38810918143, 2021

(CC BY 4.0) | ISSN 2525-3409 | DOI: http://dx.doi.org/10.33448/rsd-v10i9.18143

Tabela 2: Características sóciodemográficas das mulheres no puerpério imediato de Cesárea.

\begin{tabular}{lcc}
\hline Características sociodemográficas & N & $\%$ \\
\hline Faixa etária & & \\
$19-29$ & 24 & 80 \\
$30-39$ & 6 & 20
\end{tabular}

\section{Profissão}

Autônoma

Auxiliar operacional de loja

Administradora

26,6

Consultora de vendas

310

Estudante

26,6

Enfermeira

310

Fisioterapeuta

26,6

Professora

26,6

Outras

\section{Estado civil}

Casada

Solteira

União estavél

$9 \quad 30$

Divorciada

\section{Local onde reside}

Região metropolitana do Recife

Outros municípios de Pernambuco

Campina Grande, Paraíba

São Paulo

13,3

Lisboa, Portugal

\section{Cor}

Branca

Parda

Indígena

Negra

\section{Religião}

Católica

Evangélica

Espirita

13,3

Outras

Não segue nenhuma religião 
Research, Society and Development, v. 10, n. 9, e38810918143, 2021

(CC BY 4.0) | ISSN 2525-3409 | DOI: http://dx.doi.org/10.33448/rsd-v10i9.18143

Ensino fundamental completo

Ensino médio completo

Ensino superior completo

Ensino superior incompleto

\section{Fumante}

Sim

Não

Ingere bebida alcoólica

Sim

Não

\section{Pratica alguma atividade física?}

Sim

Não
13,3

26,7

1653,3

1136,7

$1 \quad 3,3$

$29 \quad 96,7$

1240

$18 \quad 60$

$8 \quad 26,7$

2273,3

Fonte: Autores (2021).

A Tabela 3, apresenta as características reprodutivas das mulheres, sendo assim, em relação a menarca, 26,6\% das mulheres relataram que sua primeira menstruação ocorreu entre 1998 e $2008.70 \%$ da amostra esteve grávida apenas 1 vez, $73,3 \%$ tem 1 filho vivo e 76,6\% realizou apenas 1 cesárea até o momento da pesquisa. Quanto ao uso de contraceptivos 56,7\% usam algum método, sendo a camisinha masculina o mais utilizado por cerca de 23,3\% da amostra. Em contrapartida, 23,3\% das mulheres relataram também não usar nenhum método contraceptivo. Todos os cálculos foram feitos com n=30. 
Tabela 3: Características reprodutivas das mulheres no puerpério imediato após cesárea.

\begin{tabular}{|c|c|c|}
\hline Características reprodutivas & $\mathbf{N}$ & $\%$ \\
\hline \multicolumn{3}{|l|}{ Data da menarca } \\
\hline 1998-2008 & 8 & 26,6 \\
\hline 2009-2019 & 7 & 23,3 \\
\hline Não lembra & 15 & 50 \\
\hline \multicolumn{3}{|l|}{ Quantas vezes já esteve grávida? } \\
\hline $1 \mathrm{vez}$ & 21 & 70 \\
\hline 2 vezes & 8 & 26,6 \\
\hline 3 ou + & 1 & 3,3 \\
\hline \multicolumn{3}{|c|}{ Possui quantos filhos vivos atualmente? } \\
\hline 1 filho & 22 & 73,3 \\
\hline 2 filhos & 8 & 26,6 \\
\hline \multicolumn{3}{|l|}{ Já realizou quantas cesáreas? } \\
\hline 1 cesárea & 23 & 76,6 \\
\hline 2 cesáreas & 7 & 23,3 \\
\hline \multicolumn{3}{|l|}{ Uso de contraceptivo } \\
\hline Sim & 17 & 56,7 \\
\hline Não & 13 & 43,3 \\
\hline \multicolumn{3}{|l|}{ Método contraceptivo } \\
\hline Hormônio Oral & 5 & 16,7 \\
\hline Hormônio Injetavél & 2 & 6,7 \\
\hline Preservativo Masculino & 7 & 23,3 \\
\hline Tabela & 1 & 3,3 \\
\hline DIU & 4 & 13,3 \\
\hline Coito interrompido & 4 & 13,3 \\
\hline Nenhum & 7 & 23,3 \\
\hline
\end{tabular}

Fonte: Autores (2021).

$\mathrm{Na}$ Tabela 4, foi demonstrado a média e desvio padrão dos domínios relacionados a função sexual, segundo o questionário FSFI. Em relação ao desejo (questões 1-2), 36,7\% das mulheres relataram sentir desejo ou interesse sexual poucas vezes, em menos da metade do tempo nas últimas 4 semanas. No âmbito da excitação (questões 3-6), 36,7\% das mulheres classificaram seu grau de excitação como moderado, além disso na questão de segurança quanto ao ficar excitada durante a atividade sexual, $30 \%$ relataram ter uma boa confiança, na maioria das vezes.

Quanto a lubrificação (questões 7-10), 44,8\% das puérperas informaram que ficaram lubrificadas durante a atividade sexual sempre ou quase sempre, sem sentir nenhuma dificuldade. Já com relação a ficar lubricada até o fim da relação, apenas 24,1\% relatam não ter dificuldade sempre ou quase sempre. Em relação ao orgasmo (questões 11-13), 43,3\% das voluntárias disseram conseguir atingir o clímax sempre ou quase sempre em suas relações sexuais, 39,3\% relataram, na questão 12, que não difícil chegar ao orgasmo e 36,7\% ficaram satisfeitas com seu desempenho sexual, segundo resposta dada na questão 13 . 
A satisfação sexual (questões 14-16), 53,3\% relataram estar muito satisfeitas com a intensidade de intimidade emocional com seu parceiro durante a atividade sexual levando a mais de 50\% o grau de satisfação sexual consigo, com o parceiro e com a relação nas últimas 4 semanas. Por fim, com relação a dor (questões 17-19), nas últimas 4 semanas, 26,7\% das mulheres sentiram poucas vezes dor ou desconforto durante ou após a penetração vaginal, enquanto 14,3\% sentiram dor, algumas vezes durante e após o ato sexual.

A média geral da função sexual nas mulheres do estudo foi de 24,87 , demonstrando um moderado auto relato sexual.

Tabela 4: Análise da função sexual, segundo os domínios do questionário FSFI, nas puérperas participantes do estudo.

\section{Função Sexual}

\begin{tabular}{llc}
\hline FSFI & Desejo & $3,6 \pm 0$ \\
& Excitação & $2,72 \pm 0,25$ \\
Lubrificação & $3,9 \pm 1,64$ \\
Orgasmo & $4,7 \pm 1,73$ \\
Satisfação & $4,27 \pm 1,47$ \\
Dor & $5,68 \pm 0,38$ \\
Média geral do instrumento & $24,87 \pm 1,00$ \\
\hline
\end{tabular}

Fonte: Autores (2021).

\section{Discussão}

A amostra do presente estudo contou com 30 mulheres, com faixa etária de 19 a 29 anos. Tal faixa etária também foi observada no estudo de Fernandes 2019, onde sua amostra foi de 326 mulheres, onde 51,2\% tinham idade média abaixo de 30 anos. Oliveira et al., 2017, afirma que a faixa etária ideal para gestação fica entre 21 a 30 anos pois a probabilidade de reprodução é bem maior.

Quanto ao estado civil, o estudo de Silva et al., 2018, demonstrou que dentro da sua amostra de 23 mulheres, 64,6\% eram casadas e que $77,1 \%$ da amostra total não apresentaram DSF. Fato que corrobora com o presente estudo onde $43,3 \%$ das mulheres são casadas e na média geral do FSFI observa-se que a função sexual das mulheres foi classificada como moderada.

Mathias et al., 2015, afirmou em seu estudo que as mulheres podem sofrer alterações na sua função sexual devido ao alto nível de cansaço físico e psicológico, falta de saúde, trabalho e lazer que é reflexo da baixa escolaridade referida pela amostra. Tal afirmação não corrobora com o presente estudo, visto que 53,3\% das voluntárias do estudo afirmaram possuir ensino superior completo.

Dentro da história reprodutiva, observa-se que 70\% da amostra já esteve grávida uma vez e 76,6\% teve seu primeiro filho através de uma cesárea. Isso pode ser explicado pelo fato de uma cultura da dor do parto normal, criada pela sociedade ao redor da gestante. Silva et al., 2017, mostra em seu estudo que o medo da dor, de não conseguir ter o parto, medo da conduta que pode ser realizada pelos profissionais de saúde é um dos agentes mais estressantes e colaboradores para a escolha da mulher pelo parto cesáreo, que por muitas, é visto como indolor, rápido, mais prático e seguro.

O Brasil atualmente ocupa o segundo lugar no mundo como país que realiza mais cesáreas, com uma taxa acima de $55 \%$ do total de partos que ocorre. Em toda a América Latina é a região com maior interversões, cerca de 44,3\%. A recomendação dada pela OMS é de que a taxa de partos cesáreo por país seja entre $10 \%$ a $15 \%$. O que corresponde ao percentual de nascidos que necessitam de uma cesárea para salvar sua vida (OMS, 2015). 
Com o aumento das cesáreas, o aumento das violências obstétricas sofridas também acontece. Leal 2014, afirma que em seu estudo mais de $50 \%$ das mulheres sofreram episiotomia e que $75 \%$ eram primíparas. Essa prática trás grandiosas consequências como laceração perineal grau três e quatro, infecções, hemorragias, dor a longo prazo e incontinência urinária e fecal causando a DSF em consequência de uma cesárea, muitas vezes, desnecessária (Fernandes, 2019).

As disfunções sexuais femininas após o parto podem ser reflexo dos fatores biológicos, sociais, culturais, psicológicos que as mulheres passam desde ao descobrir a gravidez até o nascimento de seu filho. Como classificação temos diversos transtornos que podem ser relacionados ao desejo, excitação, orgasmo, dor, condição clínica, afetando o puerpério das mulheres (Oliveira, 2018).

Tozo et al., 2018, afirma em seu estudo que um terço das mulheres relataram sentir falta do interesse sexual e um quarto não consegue atingir o orgasmo. Fato esse, que corrobora com o presente estudo, visto que as respostas ao questionário FSFI sobre desejo e orgasmos ficaram com média 3,6 e 4,7, respectivamente, demonstrando uma satisfação moderada ao domínios citados por parte das voluntárias. Segundo Oliveira 2018, pode ser considerado normal a redução do desejo sexual nas seis a sete primeiras semanas de pós parto, que corresponde ao puerpério, isso só passa a ser considerado anormal quando o desejo fica ausente ou reduzido constantemente e num período de tempo maior, levando a um desconforto na vida sexual da mulher.

O domínio excitação foi o que obteve menor média, de acordo com as respostas das voluntárias da pesquisa, e isso pode ser explicado muitas vezes pela falta de estimulação suficiente por parte dos seus parceiros, causando uma falta de interesse e desejo para realizar o ato sexual (Silva, 2019). Além disso, essa baixa excitação dificulta a capacidade da mulher de se manter lubrificada durante toda a relação sexual, no presente estudo 17,2\% das voluntárias relataram não conseguir ficar lubrificadas na maior parte da sua prática sexual. Toda essa alteração dificulta e em alguns casos impede o orgasmo, gerando uma baixa resposta feminina e até mesmo uma frustação com suas experiências sexuais.

No âmbito da dor, o presente estudo demonstrou que apenas 13,8\% das mulheres relataram sentir algum desconforto ou dor durante ou após o ato sexual, o que corrobora com o estudo de Siqueira 2020, onde apenas 7,29\% da amostra relatou sentir uma dor grande ou muito grande na atividade sexual.

Com relação a média obtida no questionário FSFI, a classificação do auto relato das voluntárias da pesquisa ficou como moderado, demonstrando que a mulheres não possuem DSF e retomaram suas atividades sexuais dentro do período de puerpério. Isso pode ser explicado pelo aumento progressivo de mulheres bem sucedidas no âmbito profissional e econômico na sociedade. Mulheres empoderadas para expressão seus desejos e opiniões, que vivem sem preconceitos e medo de julgamentos, vivendo o ato sexual como algo saudável e não como uma exigência ou procriação (Vieira, 2016).

\section{Conclusão}

O presente estudo evidenciou que as mulheres participantes da pesquisa que estavam no puerpério de parto cesáreo não apresentaram uma DSF e sim uma alteração moderada na atividade sexual, que esta assegurado pela literatura, pois o tempo de pós parto estava dentro do período considerado normal para volta dos desejos sexuais.

Com isso, algumas limitações ocorreram no estudo, como por exemplo, o numero da amostra que foi pequeno e a inviabilidade da realização da coleta nos hospitais da cidade do Recife, devido aos protocolos de segurança estabelecidos para barrar a proliferação da Covid-19. Mesmo com as limitações o trabalho obteve uma boa relevância quanto a evidencias cientificas geradas sobe o tema, mostrando que as DSF no puerpério do parto cesáreo não são tão frequentes ou mais frequentes que as do parto normal. 
Por fim, considera-se a partir dessa pesquisa que não há embasamentos para afirmar que as DSF são causadas pelo parto cesáreo, porém podemos observar que o retorno a vida sexual no puerpério é cada vez maior e as mulheres relatam sentir prazer e desejo sexual cada vez mais cedo.

\section{Referências}

Barreto, A. P. P., Nogueira, A., Teixeira, B., Brasil, C., Lemos, A., \& Lôrdelo, P. (2018). O impacto da disfunção sexual na qualidade de vida feminina: um estudo observacional. Revista Pesquisa em Fisioterapia, 8(4), 511-517. http://dx.doi.org/10.17267/2238-2704rpf.v8i4.2159.

Brasil. Ministério da Saúde (2013). Secretaria de Atenção à Saúde. Departamento de Atenção Básica. Caderno de Atenção Básica: saúde sexual e saúde reprodutiva. Brasília: Ministério da Saúde; https://bvsms.saude.gov.br/bvs/publicacoes/saude_sexual_saude_reprodutiva.pdf.

da Silva, N. T., \& de Oliveira Damasceno, S. (2019, April). Avaliação da satisfação sexual em universitárias. In Colloquium Vitae. ISSN: 1984-6436 (Vol. 11, No. 1, pp. 1-6). http://revistas.unoeste.br/index.php/cv/article/view/2840.

de Araujo, T. G., Scalco, S. C. P., \& Varela, D. (2019). Função e Disfunção Sexual Feminina Durante o Ciclo Gravídico-Puerperal. Revista Brasileira de Sexualidade Humana, 30(1). https://doi.org/10.35919/rbsh.v30i1.69.

de Oliveira, L. M. N., da Silva Ferreira, N. R., \& da Silva, R. M. (2017). Perfil de mulheres submetidas ao parto cesáreo em uma maternidade pública de Teresina-PI. Revista Interdisciplinar, 10(1), 37-42. https://revistainterdisciplinar.uninovafapi.edu.br/index.php/revinter/article/view/1037.

Fernandes, A. P. P. (2019). Violência obstétrica: uma análise do grau de conhecimento das gestantes e sua correlação com variáveis sócio demográficas. https://repositorio.ufu.br/handle/123456789/29713.

Leal, M. D. C., Pereira, A. P. E., Domingues, R. M. S. M., Filha, M. M. T., Dias, M. A. B., Nakamura-Pereira, M., \& Gama, S. G. N. D. (2014). Intervenções obstétricas durante o trabalho de parto e parto em mulheres brasileiras de risco habitual. Cadernos de Saúde Pública, 30, S17-S32. https://www.scielo.br/j/csp/a/gydTTxDCwvmPqTw9gTWFgGd/?format=html\&lang=pt.

de Araújo Mathias, A. E. R., Pitangui, A. C. R., Arantes, V. A., Vasconcelos, H. G., Vilela, F. M. F., \& Dias, T. G. (2015). Disfunção sexual: Avaliação de mulheres durante o terceiro trimestre gestacional. ABCS Health Sciences, 40(2). https://doi.org/10.7322/abcshs.v40i2.734.

Nunes, E. F. C., Gonçalves, B., \& Latorre, G. F. S. (2019). O papel da fisioterapia pélvica no puerpério imediato-uma revisão sistemática. Antonio Carlos Weston, 63(3), 344-348. https://www.amrigs.org.br/assets/images/upload/pdf/jornal/1580235712.pdf\#page=107.

Oliveira, T. S. D. (2018). Fatores relacionados à disfunção sexual no puerpério. https://ri.ufs.br/jspui/handle/riufs/9754.

Organização Mundial da Saúde (2015). Declaração da OMS sobre Taxas de Cesáreas. Human Reprodution Program. 1(1), 1-8. https://www.who.int/pt/publications/i/item/WHO-RHR-15.02.

Pechorro, P., Diniz, A., Almeida, S., \& Vieira, R. (2009). Validação portuguesa do índice de Funcionamento Sexual Feminino (FSFI). Laboratório de Psicologia, 7(1), 33-44. https://doi.org/10.14417/lp.684.

Pereira, T. R. C., Dottori, E. H., Mendonça, F. M. D. A. F., \& Beleza, A. C. S. (2018). Avaliação da função sexual feminina no puerpério remoto: um estudo transversal. Revista Brasileira de Saúde Materno Infantil, 18, 289-294. https://www.scielo.br/j/rbsmi/a/x6kkkLHHs36Q3pb9hNGHTPq/?lang=pt.

Santos, L. M. S. S., Silva, M. R. G., Latorre, G. F. S., \& Jorge, L. B. (2019). Tratamento da disfunção sexual feminina através da utilização de dilatadores vaginais. Revista da AMRIGS,63, 85-88. https://www.researchgate.net/profile/Victor-Kuiava/publication/334465512_Complicacoes_de_um a_rara_doenca_dermatomiosite/links/5d2c776b299bf1547cb824bd/Complicacoes-de-uma-rara-doenca-dermatomiosite.pdf\#page=87.

Silva, A. C. L., Félix, H. C. R., Ferreira, M. B. G., Wysocki, A. D., Contim, D., \& Ruiz, M. T. (2017). Preferência pelo tipo de parto, fatores associados à expectativa e satisfação com o parto. Revista Eletrônica de Enfermagem, 19. https://www.revistas.ufg.br/fen/article/view/44139.

Silva, R. E. D. L., \& Bernardo, S. H. S. (2017). Prevalência das disfunções sexuais em mulheres no pós-parto em um hospital escola no Recife, Pernambuco. http://tcc.fps.edu.br:80/jspui/handle/fpsrepo/18.

Silva, T. B., de Bulhões, T. R. B., Cirqueira, R. P., \& Ferreira, J. B. (2018). Análise da função sexual e imagem genital em primíparas e multíparas pós-parto vaginal. ID on line REVISTA DE PSICOLOGIA, 12(39), 97-111. https://idonline.emnuvens.com.br/id/article/view/982.

Siqueira, E. M. M., \& Falcao, V. S. (2020). Saúde da mulher e as disfunções sexuais em acadêmicas do ensino superior (Doctoral dissertation). http://repositorio.fametro.com.br/jspui/handle/123456789/699.

Tozo, I. M., Lima, S. M. R. R., Gonçalves, N., de Moraes, J. C., \& Aoki, T. (2018). Disfunção sexual feminina: a importância do conhecimento e do diagnóstico pelo ginecologista. Arquivos Médicos dos Hospitais e da Faculdade de Ciências Médicas da Santa Casa de São Paulo, 52(3), 94-99. http://arquivosmedicos.fcmsantacasasp.edu.br/index.php/AMSCSP/article/view/447.

Vieira, K. F. L., Nóbrega, R. P. M. D., Arruda, M. V. S., \& Veiga, P. M. D. M. (2016). Representação social das relações sexuais: um estudo transgeracional entre mulheres. Psicologia: Ciência e Profissão, 36, 329-340. https://www.scielo.br/j/pcp/a/tnnBmB6vVRFvNNsPxxHtNVs/abstract/?lang=pt.. 